\title{
Herpes simplex virus type 1 VP22-mediated intercellular delivery of PTEN increases the antitumor activity of PTEN in esophageal squamous cell carcinoma cells in vitro and in vivo
}

\author{
XIAN YU ${ }^{1 *}$, TINGTING LI $^{2 *}$, YIFAN XIA ${ }^{3}$, JUN LEI $^{2}$, YAN WANG $^{2}$ and LIJUAN ZHANG ${ }^{2}$ \\ ${ }^{1}$ Department of Pharmacy, The Second Affiliated Hospital, Chongqing Medical University, Chongqing 400010; \\ ${ }^{2}$ Institute of Materia Medica, North Sichuan Medical College, Nanchong, Sichuan 637007; \\ ${ }^{3}$ Department of Orthopaedics, Chongqing General Hospital, Chongqing 400014, P.R. China
}

Received November 30, 2015; Accepted January 12, 2016

DOI: 10.3892/or.2016.4694

\begin{abstract}
In the past decade, studies have revealed that the phosphatase and tensin homolog (PTEN) protein, a tumor suppressor, comprises a potential biological marker and therapeutic target for esophageal squamous cell carcinoma (ESCC). As such, the delivery of the PTEN gene represents a powerful strategy for ESCC therapy. The tegument protein VP22 of herpes simplex virus type 1 (HSV-1) has been reported to act as a transporter of heterologous proteins across the host cell membrane, thereby enhancing the biological functions of these proteins. In the present study, the intercellular delivery and antitumor activity of the fusion protein PTEN-VP22 were examined in the esophageal squamous cell carcinoma cell line Eca109 both in vitro and in vivo. VP22-mediated PTEN intercellular delivery was confirmed in the Eca109 cells by western blot analysis and by quantitation of immunofluorescence. VP22 alone did not exert antiproliferative effects or induce cell cycle arrest, induction of apoptosis, blockage of the Akt and focal adhesion kinase (FAK) pathways, tumor growth inhibition, or antiangiogenic effects in Eca109 cells. However, compared with PTEN alone, PTEN-VP22 exerted significantly higher antiproliferative effects and induced cell cycle arrest at G1 stage, apoptosis and antiangiogenic effects in Eca109 cells. Together, our findings demonstrate that VP22 alone does not exert antitumor activity directly; however, this protein mediates the intercellular delivery of PTEN and thereby increases its intracellular concentration to achieve a therapeutic steady state, leading to an overall increase in the antitumor activity
\end{abstract}

Correspondence to: Dr Xian Yu, Department of Pharmacy, The Second Affiliated Hospital, Chongqing Medical University, 76 Linjiang Road, Chongqing 400010, P.R. China

E-mail: xiannyyu@gmail.com

${ }^{*}$ Contributed equally

Key words: phosphatase and tensin homolog, VP22, Eca109, intercellular delivery, gene therapy of PTEN. This study provides further experimental data to confirm the potential of VP22-based intercellular delivery strategies for enhancing the efficacy of gene therapy for cancer treatment.

\section{Introduction}

Esophageal cancer is one of the most common fatal types of cancer worldwide. In China, esophageal cancer ranks as the fourth leading cause of cancer-related mortality. Esophageal squamous cell carcinoma (ESCC) and esophageal adenocarcinoma are the two major histological types of esophageal carcinoma (1), and ESCC is the main histological subtype in the so-called Asian belt, which includes Turkey, northeastern Iran, Kazakhstan and northern and central China (2). Although several strategies have been developed for the treatment of ESCC, including surgery, chemotherapy, radiation and comprehensive treatment, the 5-year overall survival rate for ESCC remains as low as $20-30 \%$ (3). Therefore, more effective and targeted therapies for ESCC are urgently needed.

Loss of the phosphatase and tensin homolog (PTEN) protein, a tumor suppressor, has been reported as a prognostic factor in numerous types of cancer such as endometrial carcinomas, gliomas, prostate carcinomas, gastric cancers, lung and breast cancers (4-9). Recent studies have revealed that the expression of PTEN is correlated with prognosis in patients with ESCC (10-12). Increased activity of the PTEN/PI3K/Akt signaling pathway was found to inhibit ESCC cell proliferation both in vitro and in vivo $(13,14)$. Conversely, ESCC cell proliferation is enhanced by transfection with a PTEN antisense oligonucleotide (15). Therefore, delivery of the tumor suppressor gene PTEN represents a powerful strategy for ESCC therapy. However, to date, there have been few reports of PTEN transfer-based gene therapies for the treatment of ESCC. A previous study demonstrated that the adenovirusmediated transfer of PTEN inhibits the growth of esophageal cancer cells in vitro and in vivo (16). Since virus-mediated gene therapy is associated with safety problems, the use of non-viral vectors for cancer gene therapy is a potential alternative. Of these, cell-penetrating peptides (CPPs) have attracted considerable attention for their potential utility in the delivery of 
exogenous molecules into living cells. Tegument protein VP22 of herpes simplex virus type 1 (HSV-1) is a CPP that is capable of transporting heterologous proteins, such as p53, p27, cytosine deaminase, and Hsp70, across the cell membrane, thereby enhancing the biological functions of these proteins (17-20). However, the mechanisms involved in the delivery of these proteins by VP22 have not been fully characterized.

We previously reported that VP22-mediated intercellular delivery of PTEN enhances the antitumor effects of PTEN in the breast tumor cell line BT549 (21). In the present study, the intercellular delivery and the antitumor activity of the fusion gene PTEN-VP22 were examined in an ESCC cell line both in vitro and in vivo.

\section{Materials and methods}

Cell culture. Human esophageal carcinoma cells (Eca109) were cultivated in Dulbecco's modified Eagle's medium (DMEM; Gibco-Life Technologies, Carlsbad, CA, USA) supplemented with $10 \%$ bovine serum (FBS; Gibco), $100 \mathrm{U} / \mathrm{ml}$ penicillin and $100 \mathrm{mg} / \mathrm{ml}$ streptomycin at $37^{\circ} \mathrm{C}$ with $5 \% \mathrm{CO}_{2}$.

Eukaryotic expression vector construction. The pcDNA3VP22, pcDNA3-PTEN and pcDNA3-PTEN-VP22 vectors were utilized in the present study for the expression of wildtype human PTEN protein, HSV-1 VP22 protein, and the N-terminal VP22-fused PTEN protein (PTEN-VP22), respectively, as previously described (21).

Cell transfection. Eca109 cells were grown to $70 \%$ confluency and washed twice with phosphate-buffered saline (PBS). Washed cells were transfected with the plasmids diluted in serum-free DMEM containing Lipofectamine 2000 reagent (Invitrogen-Life Technologies, Waltham, MA, USA), according to the manufacturer's instructions. Prior to the start of the experiment, transfection efficiency was determined using pSV- $\beta$-galactosidase (Promega, Madison, WI, USA).

Western blot analysis. Eca109 cells were transfected with $5 \mu \mathrm{g} / \mathrm{ml}$ of pcDNA3, pcDNA3-PTEN, pcDNA3-PTEN-VP22, or pcDNA3-VP22. After $48 \mathrm{~h}$, total protein was extracted from the cells. Protein concentrations were determined using the Bradford assay (Bio-Rad Laboratories, Inc., Hercules, CA, USA). Proteins were separated by $10 \%$ SDS-PAGE and immunoblotting was performed using primary antibodies specific to PTEN (Santa Cruz Biotechnology, Inc., Dallas, TX, USA), phospho-Akt (Ser473; Cell Signaling Technology, Inc., Danvers, MA, USA), or phospho-FAK (Tyr576/577; Cell Signaling Technology). Horseradish peroxidaseconjugated anti-IgG (Santa Cruz Biotechnology) was used as the secondary antibody for the DAB detection system (Wuhan Boster Biological Technology, Ltd., Wuhan, China). Antibodies specific to total Akt (Cell Signaling Technology), total FAK (Cell Signaling Technology), or $\beta$-actin (Wuhan Boster Biological Technology) were used as loading controls. Each test was repeated in triplicate.

Immunofluorescence quantitation. Eca109 cells were transfected with $5 \mu \mathrm{g} / \mathrm{ml}$ of each plasmid. After $48 \mathrm{~h}$, cells were fixed in cold methanol for $10 \mathrm{~min}$ at $25^{\circ} \mathrm{C}$ and then permeabi- lized by incubation with $0.2 \%$ Triton $\mathrm{X}-100$ for 90 min at $25^{\circ} \mathrm{C}$. After blocking for $30 \mathrm{~min}$ in $5 \%$ non-fat milk at $25^{\circ} \mathrm{C}$, cells were incubated with rabbit anti-PTEN antibody (Santa Cruz Biotechnology) at $4^{\circ} \mathrm{C}$ overnight. After three washes, cells were incubated with fluorescein isothiocyanate-conjugated sheep anti-rabbit IgG (Santa Cruz Biotechnology) for $1 \mathrm{~h}$ at $25^{\circ} \mathrm{C}$ and analyzed using a Fluoroskan Ascent FL microplate reader (Thermo Fisher Scientific, Waltham, MA, USA). Each test was repeated in triplicate.

Analysis of cell proliferation by Cell Counting kit-8 assay. Eca109 cells were transfected with $10 \mu \mathrm{g} / \mathrm{ml}$ of pcDNA3 and varying concentrations $(2,4,6,8$ or $10 \mu \mathrm{g} / \mathrm{ml})$ of pcDNA3VP22, pcDNA3-PTEN or pcDNA3-PTEN-VP22. Different quantities of pcDNA3 were added to each well to ensure that the same overall amount of DNA was present in every well. Transfected cells were harvested at $10 \mathrm{~h}$ and plated in 96-well plates (Corning Life Sciences, Tewksbury, MA, USA) at a density of 3,000 cells/well for each treatment condition. At 24, 48 and $72 \mathrm{~h}$ after transfection, $10 \mu \mathrm{l}$ of WST- 8 dye (Beyotime Institute of Biotechnology, Shanghai, China) was added to each well. Plates were incubated at $37^{\circ} \mathrm{C}$ for $1 \mathrm{~h}$ and the absorbance (A) at $450 \mathrm{~nm}$ was measured using an iMark bio-microplate reader (Bio-Rad Laboratories). Cell survival was determined as $A_{\text {treated }} / A_{\text {control }}$. Each test was repeated in triplicate.

Cell cycle analysis. Eca109 cells were transfected with $6 \mu \mathrm{g} / \mathrm{ml}$ of each vector. Forty-eight hours later, cells were harvested by trypsinization, fixed with $70 \%$ ethanol and stored at $-20^{\circ} \mathrm{C}$ overnight. Cell nuclei were stained by incubation for $30 \mathrm{~min}$ with $0.2 \mathrm{mg} / \mathrm{ml} \mathrm{RNase}$ solution (Beyotime Institute of Biotechnology) and $0.05 \mathrm{mg} / \mathrm{ml}$ propidium iodide (PI). Analysis was performed using a FACSVantage SE flow cytometer (BD Biosciences, Franklin Lakes, NJ, USA). Each test was repeated in triplicate.

Analysis of apoptosis by Annexin V/PI double staining. Eca109 cells were transfected with $6 \mu \mathrm{g} / \mathrm{ml}$ of each vector. Forty-eight hours later, cells were double stained using an Annexin V/PI apoptosis detection kit (Beyotime Institute of Biotechnology), according to the manufacturer's instructions. Cell apoptosis was then evaluated using a FACSVantage SE flow cytometer (BD Biosciences). Each test was repeated in triplicate.

Animal experiments. The present study was carried out in accordance with the Guide for the Care and Use of Laboratory Animals (2011). All animal experiments were conducted in the Experimental Animal Center of Chongqing Medical University. The use of animals and the experimental protocols were approved by the Animal Care and Use Committee of Chongqing Medical University. Female 4- to 6-week-old BALB/c nude mice (Animal Laboratory Center of Chongqing Medical University) were maintained in specific pathogenfree, environmentally controlled conditions on standard laboratory chow. Eca109 cells were suspended in serum-free medium at a density of $1 \times 10^{7} / \mathrm{ml}$, and $0.1 \mathrm{ml}$ of the suspension was injected subcutaneously into the dorsal area of each nude mouse. Thirty-two tumor-bearing mice were divided randomly into four groups ( $n=8 /$ group), and treatments were carried out when the tumors had grown to $6 \mathrm{~mm}$ in diameter. 
A mixture of $100 \mu \mathrm{g}$ of the vector and $100 \mu \mathrm{l}$ of Lipofectamine 2000 reagent (Invitrogen Life Technologies) were injected intratumorally into each mouse from each group, followed by another injection the next day. One group was injected with pcDNA3 and was designated the control group. The other groups were treated with pcDNA3-PTEN, pcDNA3-VP22, or pcDNA3-PTEN-VP22. Tumor growth was measured using calipers every other day after the second injection, and tumor volume was calculated using the following formula: Tumor volume $\left(\mathrm{mm}^{3}\right)=$ length $\mathrm{x}$ width ${ }^{2} \times 0.5$. After 15 days, the mice were sacrificed using diethyl ether and tumor specimens were harvested for western blot or immunohistochemical analysis.

Microvessel counting by immunohistochemistry. Tumor tissue specimens were formalin-fixed and paraffin-embedded (FFPE). Sections from FFPE tissues were subjected to immunohistochemical staining according to a standard method. Briefly, $4-\mu \mathrm{m}$ thick sections were obtained using a microtome, transferred onto adhesive slides and dried at $59^{\circ} \mathrm{C}$ for $60 \mathrm{~min}$. After deparaffinization and rehydration, the sections were treated with a $3 \%$ hydrogen peroxide solution for $10 \mathrm{~min}$ to block endogenous peroxidase and then pretreated for antigen retrieval in $10 \mathrm{mM}$ citrate buffer ( $\mathrm{pH}$ 6.0) in a microwave oven for $20 \mathrm{~min}$. Tissue sections were incubated at room temperature for $1 \mathrm{~h}$ with a CD31-specific antibody (Santa Cruz Biotechnology) and then a horseradish peroxidase-labeled anti-immunoglobulin for $30 \mathrm{~min}$. Sections were then developed with 3,3'-diaminobenzidine. To quantify the microvessel density of the tumor sections, the microvessels in three randomly selected fields of one randomly selected section of each mouse were counted at a magnification of x20 (Eclipse 50i microscope; Nikon, Tokyo, Japan). Every single brownstained cell and cell cluster was calculated as a blood vessel.

Statistical analysis. Data are expressed as means \pm standard error of the mean (SEM). Statistical analysis was performed across multiple groups using analysis of variance (ANOVA) and confirmed between individual groups using the StudentNewman-Keuls method. $\mathrm{P}<0.05$ was considered statistically significant.

\section{Results}

VP22 mediates PTEN intercellular delivery in Eca109 cells. We previously reported that VP22 mediates the intercellular delivery of PTEN in the breast tumor cell line BT549 (21). In the present study, trafficking of the PTEN-VP22 fusion protein was examined in Eca109. Western blot analysis showed that the pcDNA3-PTEN-transfected cells exhibited high levels of PTEN expression $(\sim 60 \mathrm{kDa})$, and that pcDNA3-PTEN-VP22-transfected cells exhibited higher expression of PTEN-VP22 ( 90 kDa). Except for those transfected with pcDNA3-PTEN, each of the cell populations expressed very low endogenous levels of PTEN at $48 \mathrm{~h}$ after treatment (Fig. 1A). Plasmid-transfected Eca109 cells were observed by immunofluorescence microscopy and the levels of PTEN expression were determined by fluorescence quantitation (Fig. 1B). At $48 \mathrm{~h}$ after transfection, there was no difference in PTEN-specific fluorescence between the pcDNA3 (negative control)- and the pcDNA3-VP22-transfected cells. High

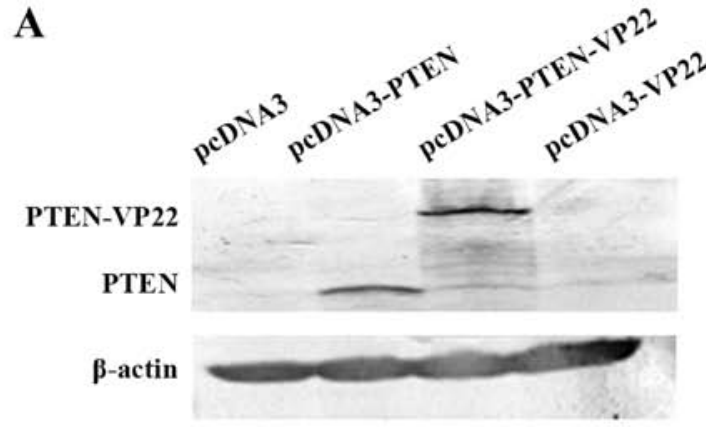

B
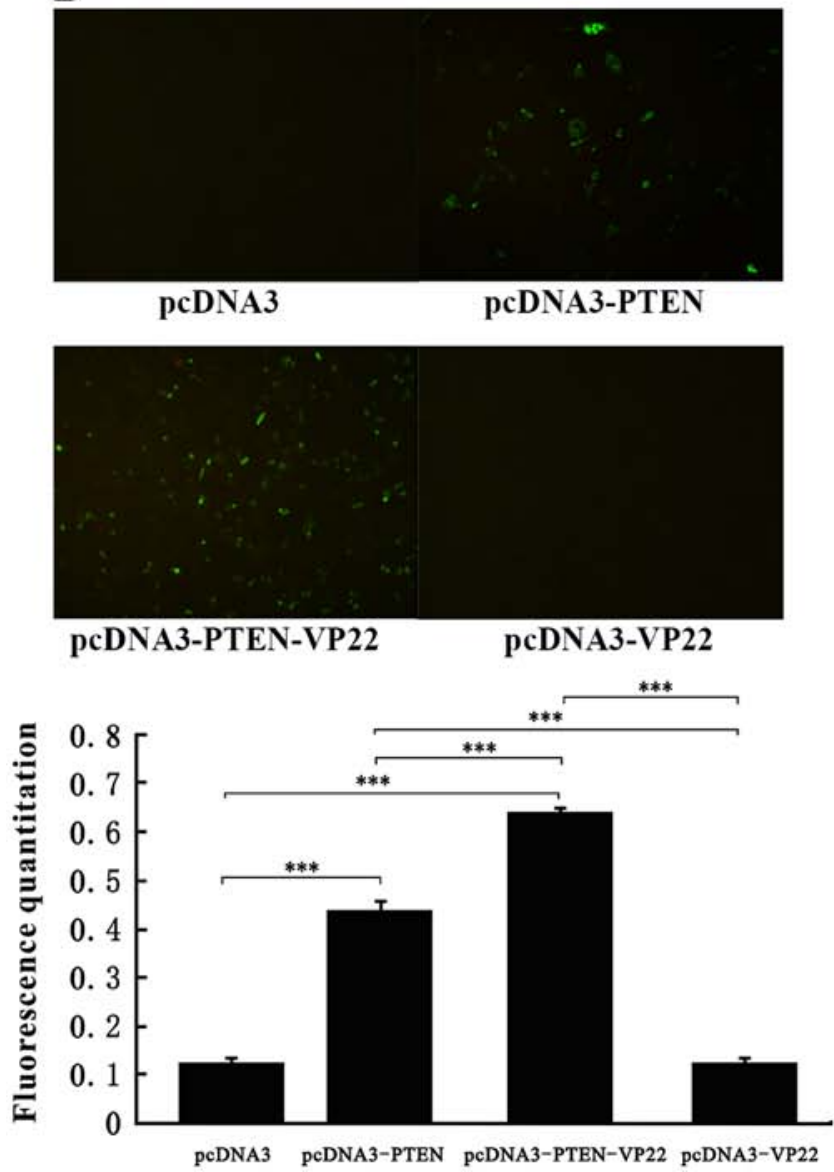

Figure 1. VP22 mediates PTEN intercellular trafficking in Eca109 cells. (A) PTEN-VP22 fusion protein expression in Eca109 cells at $48 \mathrm{~h}$ after transfection. (B) Fluorescence quantitation of PTEN-VP22 and PTEN in Eca109 cells using an anti-PTEN antibody at $48 \mathrm{~h}$ after transfection. ${ }^{* * *} \mathrm{P}<0.001$.

levels of fluorescence were detected in the pcDNA3-PTEN $(\mathrm{P}<0.001$ vs. pcDNA3) and pcDNA3-PTEN-VP22 $(\mathrm{P}<0.001$ vs. pcDNA3) groups. Additionally, the pcDNA3-PTENVP22 group exhibited a higher level of fluorescence than the pcDNA3-PTEN group $(\mathrm{P}<0.001)$. These results suggest that VP22 may mediate the intercellular delivery of PTEN, resulting in increased distribution of the latter within Eca109 cells.

VP22 enhances PTEN-mediated antiproliferative activity in Eca109 cells. Eca109 cells were transfected with various doses $(2,4,6,8$ and $10 \mu \mathrm{g} / \mathrm{ml})$ of pcDNA3-PTEN, pcDNA3PTEN-VP22 or pcDNA3-VP22, and cell survival rates were evaluated at 24, 48 and $72 \mathrm{~h}$ after transfection. As shown in 

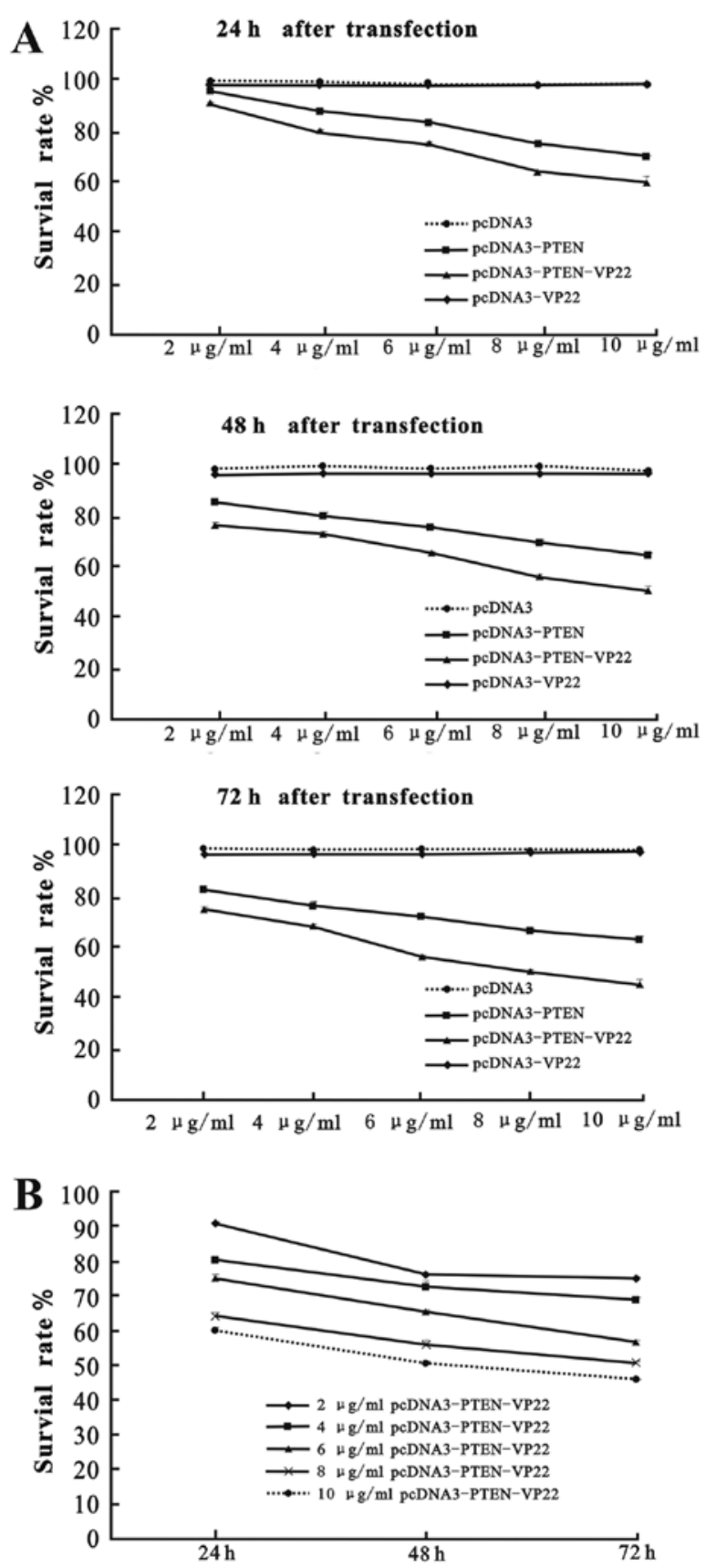

Figure 2. VP22 enhances PTEN-mediated antiproliferative activity in Eca109 cells. (A) Dose-response curve for the antiproliferative activity of pcDNA3-PTEN-VP22 in Eca109 cells. (B) Time-response curve showing the antiproliferative activity of pcDNA3-PTEN-VP22 in Eca109 cells. Cell survival was determined as $\mathrm{A}_{\text {treated }} / \mathrm{A}_{\text {control }}$.

Fig. 2, there was no difference in the cell survival rates of the pcDNA3 and the pcDNA3-VP22 group $(\mathrm{P}>0.05)$, indicating that VP22 alone did not exert antiproliferative effects on Eca109 cells. In contrast, cell proliferation was inhibited in the pcDNA3-PTEN and pcDNA3-PTEN-VP22 groups in a doseand time-dependent manner. Indeed, both pcDNA3-PTEN and pcDNA3-PTEN-VP22 exhibited a significantly greater antiproliferative activity compared with pcDNA3 at identical concentrations $(2-10 \mu \mathrm{g} / \mathrm{ml})$ at 24,48 and $72 \mathrm{~h}$ post-transfection $(\mathrm{P}<0.001$; Fig. 2A). Furthermore, the efficacy of inhibition of proliferation by pcDNA3-PTEN-VP22 was greater than that of pcDNA3-PTEN $(\mathrm{P}<0.001$ at the same concentrations at
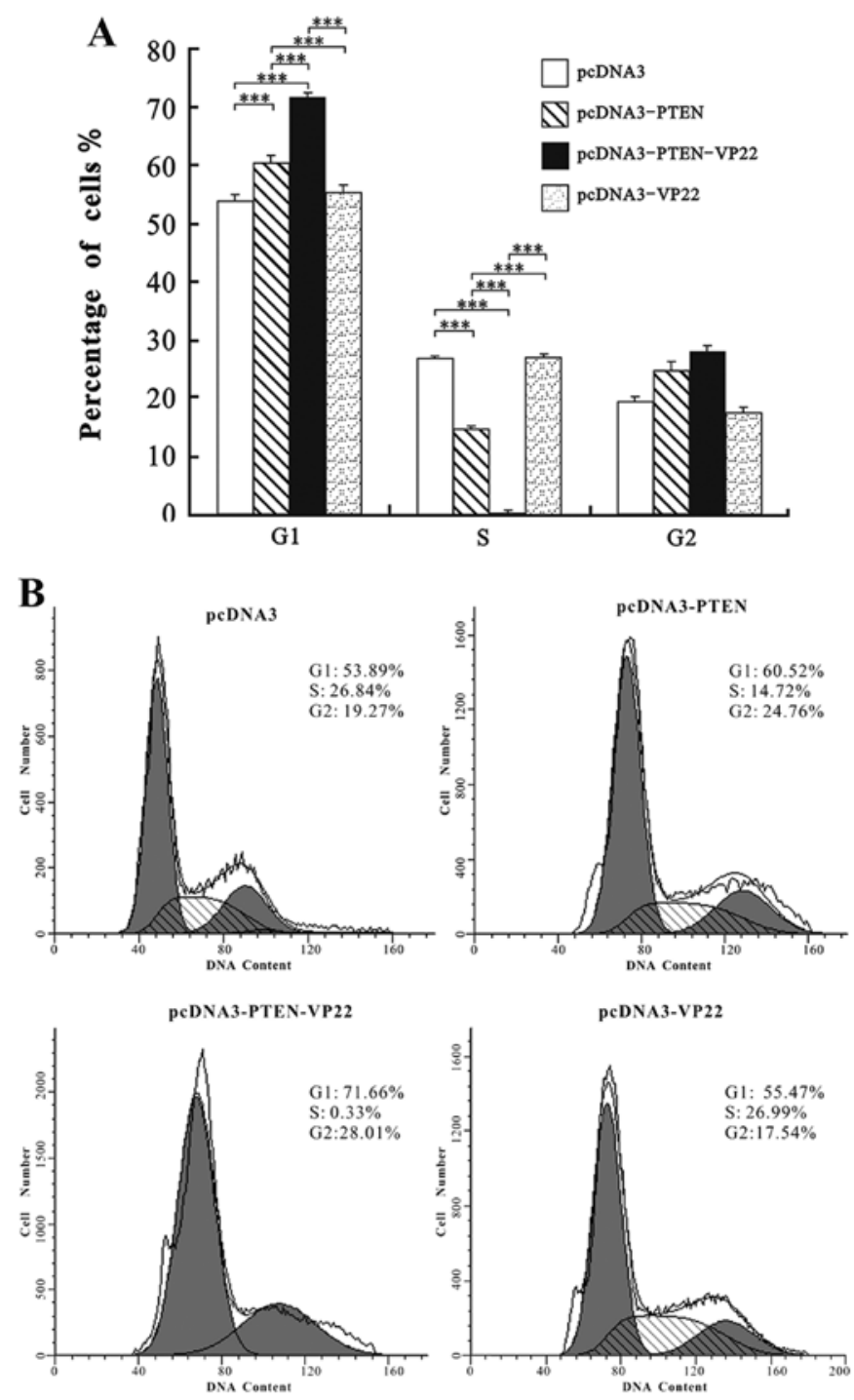

Figure 3. VP22 enhances PTEN-mediated cell cycle arrest at G1 in Eca109 cells. (A) Cell cycle analysis of Eca109 cells at $48 \mathrm{~h}$ post-transfection. (B) Results from one representative experiment are shown. ${ }^{* * * *} \mathrm{P}<0.001$.

24, 48 and 72 h; Fig. 2A). Higher concentrations of pcDNA3PTEN-VP22 were found to yield significantly lower levels of Eca109 proliferation than lower concentrations $(2-10 \mu \mathrm{g} / \mathrm{ml})$ at each time-point $(\mathrm{P}<0.001 ; \mathrm{Fig}$. 2B). Therefore, these results suggest that VP22 enhances the antiproliferative activity of PTEN in Eca109 cells.

VP22 enhances PTEN-mediated cell cycle arrest at G1 phase in Eca109 cells. The cell cycle distribution of Eca109 cells was examined at $48 \mathrm{~h}$ after transfection with $6 \mu \mathrm{g} / \mathrm{ml}$ of each plasmid. As shown in Fig. 3, the cell cycle distribution of the pcDNA3 group did not differ from that of the pcDNA3-VP22 group ( $\mathrm{P}>0.05)$, indicating that VP22 alone did not affect cell cycle progression in Eca109 cells. Compared with pcDNA3, treatment with pcDNA3-PTEN and pcDNA3-PTEN-VP22 resulted in significant increases in the percentage of cells in G1 phase $(\mathrm{P}<0.001)$ and a significant concomitant decrease in the number of cells in the $\mathrm{S}$ phase $(\mathrm{P}<0.001$; Fig. 3). However, pcDNA3-PTEN-VP22 induced higher rates of G1 arrest than pcDNA3-PTEN $(\mathrm{P}<0.001)$. These findings suggest that VP22 

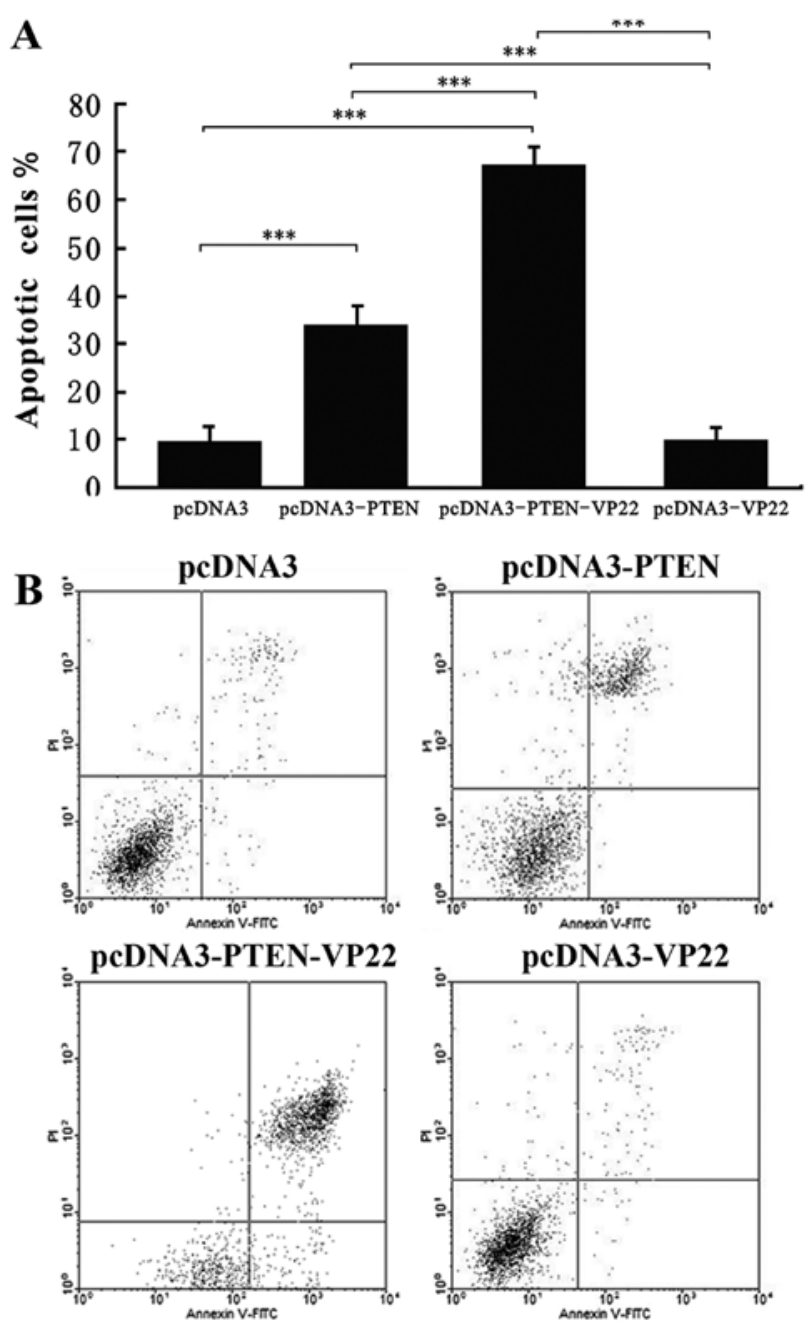

Figure 4. VP22 enhances PTEN-mediated apoptotic induction in Eca109 cells. (A) The percentage of apoptotic cells was compared between all groups at $48 \mathrm{~h}$ after transfection. (B) Results from one representative experiment are shown. ${ }^{* * *} \mathrm{P}<0.001$.

enhances the rate of PTEN-mediated G1 cell cycle arrest in Eca109 cells.

VP22 enhances PTEN-mediated apoptotic induction in Ecal09 cells. To determine whether the antitumor activity of PTEN-VP22 in Eca109 cells occurs via the induction of apoptosis, cells were stained with Annexin V and PI and visualized by fluorescence photomicrography. Cells were examined at $48 \mathrm{~h}$ after transfection with $6 \mu \mathrm{g} / \mathrm{ml}$ of various plasmids. Compared with pcDNA3 (the negative control), pcDNA3-VP22 did not induce higher levels of apoptosis; however, the rate of apoptosis of cells transfected with pcDNA3-PTEN differed significantly from those transfected with pcDNA3 $(\mathrm{P}<0.001$; Fig. 4). In addition, a significant increase in apoptosis was detected in cells transfected with pcDNA3-PTEN-VP22 vs. pcDNA3-PTEN $(\mathrm{P}<0.001)$, indicating that VP22 enhances PTEN-mediated induction of apoptosis in Eca109 cells.

VP22 enhances PTEN-mediated decrease in the level of phosphorylated Akt and FAK in Ecal09 cells. Depending on the specific plasmids transfected, we observed different levels of Akt phosphorylation at Ser473 and FAK phosphorylation

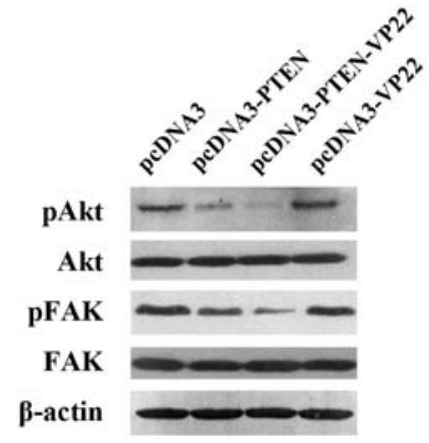

Figure 5. VP22 enhances the PTEN-mediated decrease in the levels of phosphorylated Akt and FAK.

at Tyr576/577 (Fig. 5). Phospho-Akt and phospho-Fak were highly expressed in the pcDNA3 pcDNA3-VP22 groups. In contrast, these proteins were expressed at low levels in the pcDNA3-PTEN group and at very low levels in the pcDNA3PTEN-VP22 group. These results indicate that VP22 alone does not affect the levels of phosphorylated Akt and FAK, but enhances the PTEN-mediated decrease in the levels of phospho-Akt and phospho-FAK.

VP22 enhances PTEN-mediated antitumor efficacy in vivo. Results from our present in vitro experiments indicate that PTEN-VP22 and PTEN exert antitumor effects on Eca109 cells in vitro. To further these studies, we examined the antitumor effects of PTEN-VP22 in vivo using a transplanted tumor nude mouse model. Exposure of mice to pcDNA3-PTEN-VP22 and pcDNA3-PTEN resulted in significant suppression of tumor growth over a 15 -day observation period $(\mathrm{P}<0.01)$, compared with the control group (Fig. 6A). Additionally, significant suppression of tumor growth was detected in the pcDNA3PTEN-VP22-treated mice vs. the pcDNA3-PTEN-treated mice $(\mathrm{P}<0.01)$. However, no obvious difference in tumor growth was observed between the pcDNA3-VP22-treated mice and the control mice $(\mathrm{P}>0.05)$. Additionally, we examined the antiangiogenic effect of PTEN-VP22 on Eca109 tumors. CD31 was used to analyze microvessel density. CD31-positive vessels were abundant in pcDNA3- and pcDNA3-VP22treated Eca109 tumors $(\mathrm{P}>0.05)$. In contrast, the microvessel density was significantly decreased in the pcDNA3-PTENand pcDNA3-PTEN-VP22-treated Eca109 tumors $(\mathrm{P}<0.01$; Fig. 6B), and was much lower in the pcDNA3-PTEN-VP22treated tumors than in those treated with pcDNA3-PTEN $(\mathrm{P}<0.01)$. These findings demonstrate that VP22 alone does not exert antitumor effects, but that VP22 may enhance PTENmediated antitumor efficacy in vivo.

\section{Discussion}

Research during the past decade has demonstrated that PTEN comprises a potential biological marker and therapeutic target for ESCC (10-15). However, since the efficiency and targeting of gene transfer approaches remain low, there have been only a few reports on PTEN gene therapy for the treatment of ESCC. Regardless, CPPs that are capable of carrying large macromolecules across cellular membranes with high efficiency and minimal toxicity have recently been found to overcome the 

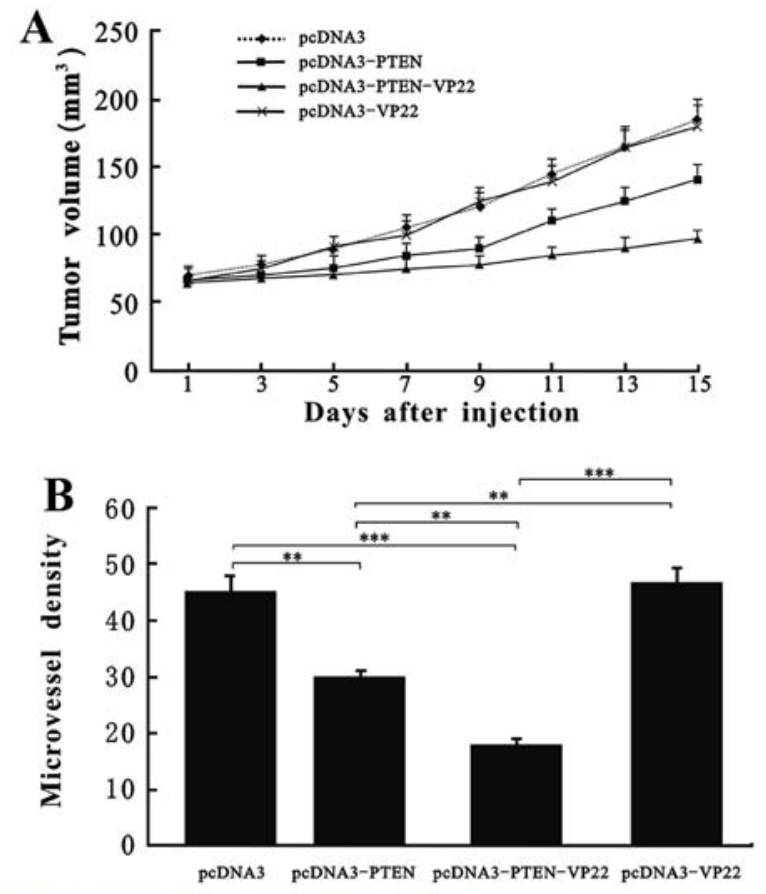

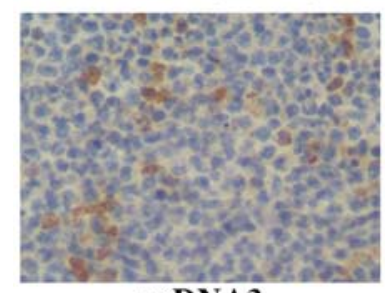

pcDNA3

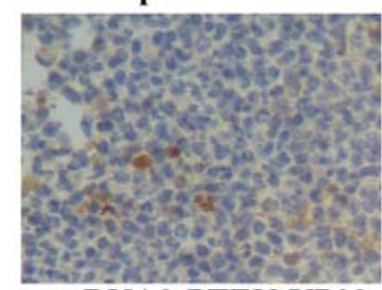

pCDNA3-PTEN-VP22
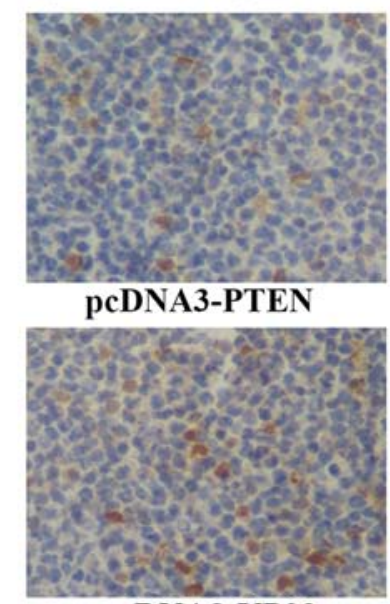

pcDNA3-VP22
pCDNA3-PTEN

Figure 6. VP22 enhances PTEN-mediated antitumor efficacy in Eca109bearing mice in vivo. (A) Tumor volumes were compared between all groups. (B) Effect of all groups on microvessel density in Eca109 tumors Representative sections (final magnification, $\mathrm{x} 200$ ) are shown for each of the four treatment groups (pcDNA3, pcDNA3-VP22, pcDNA3-PTEN and pcDNA3-PTEN-VP22). Five fields per slide per treatment group were examined. ${ }^{* *} \mathrm{P}<0.01,{ }^{* * *} \mathrm{P}<0.001$.

cell membrane impermeability issues associated with gene transfer (22).

VP22 interacts with the cell membrane more easily in a partially unfolded state (23). The motif responsible for the ability of the HSV-1 VP22 protein to penetrate the cell membrane lies in the C-terminal 34-amino acids 'DAA TATRGRSAASR PTERPRAPARSASRPRRPVD’ (24). Additionally, our previous findings (21) demonstrated that the C-terminus of VP22 is essential for intercellular delivery. In this study, expression vectors for the wild-type PTEN gene and the PTEN-VP22 fusion gene were constructed and their antitumor activities were compared in the Eca109 ESCC cell line with low endogenous expression of PTEN (Fig. 1).

High expression levels of PTEN and higher expression levels of the PTEN-VP22 fusion protein were observed in the
pcDNA3-PTEN group and the pcDNA3-PTEN-VP22 group, respectively (Fig. 1A). Furthermore, the pcDNA3-PTEN-VP22 group exhibited a higher level of PTEN fluorescence than the pcDNA3-PTEN group. The results of our previous study demonstrated (21) that VP22 transports PTEN across the cell membrane in BT549 cells with a typical VP22 pattern (primary transfected cells with cytoplasmic and nuclear staining surrounded by recipient cells with nuclear staining), resulting in a wider distribution, without affecting the subcellular localization of this protein. These results suggest that VP22 may increase PTEN distribution via intercellular delivery in Eca109 cells as well. Several reports have indicated that proliferation of ESCC cells is inhibited via increased PTEN activity both in vitro and in vivo $(13,14)$. In this study, to demonstrate whether the VP22-mediated increase in PTEN distribution enhances the antitumor activity of PTEN in Eca109 cells, we investigated the effects of PTEN-VP22 on the behavior of Eca109 cells both in vitro and in vivo. The PTEN-VP22 fusion protein induced a stronger, time- and dose-dependent antiproliferative effect than PTEN alone in vitro (Fig. 2). Conversely, VP22 did not display antiproliferative effects in Eca109 cells (Fig. 2A), suggesting that the observed increase in antiproliferative activity is due to the delivery of PTEN by VP22.

To elucidate the mechanisms by which PTEN-VP22 induces these antitumor effects, we investigated whether higher levels of protein delivery were correlated with cell cycle arrest or increased apoptotic activity in Eca109 cells. Cell cycle analysis demonstrated that treatment with PTENVP22 resulted in enhanced cell cycle arrest at G1 phase compared with PTEN. Conversely, VP22 did not alter the cell cycle distribution in Eca109 cells (Fig. 3). Previous studies have shown that transduction of the wild-type PTEN gene into cancer cells induces apoptosis $(25,26)$. Here, we observed that PTEN-VP22 enhanced apoptosis relative to PTEN alone, whereas VP22 had no effect on apoptosis levels (Fig. 4). These data suggest that VP22 enhances PTEN-mediated G1 cell cycle arrest and apoptosis in Eca109 cells, which may be induced by the VP22-mediated intercellular distribution of PTEN.

PTEN is involved in the regulation of a variety of signal transduction pathways, e.g. PTEN suppresses the PI3K/Akt pathway via dephosphorylation of PIP3 (phosphatidylinositol 3,4,5-triphosphate) (27), thereby inhibiting tumor proliferation and migration and inducing apoptosis $(28,29)$. PTEN is also involved in the dephosphorylation and inactivation of FAK, thereby additionally regulating tumor angiogenesis, migration, invasion and metastasis $(30,31)$. In the present study, we investigated whether higher PTEN delivery levels were correlated with decreased levels of phosphorylated Akt or FAK in Eca109 cells. The phospho-Akt and phospho-FAK levels were significantly lower in the presence of PTEN-VP22 than of PTEN in Eca109 cells, but were not altered by VP22 alone (Fig. 5). Together, these data demonstrate that VP22 enhances PTEN-mediated suppression of the PI3K/Akt and FAK pathways, consequently enhancing the antitumor activity of PTEN.

Finally, to empirically investigate the inhibitory effect of PTEN-VP22 on tumor growth directly, we developed an in vivo tumor model involving the intratumoral administration of pcDNA3, pcDNA3-PTEN, pcDNA3-PTEN-VP22 or pcDNA3-VP22. While treatment with PTEN alone resulted in 
moderate inhibition of tumor growth and angiogenic effects in Eca109-bearing mice, PTEN-VP22 markedly suppressed tumor growth and exerted strong angiogenic effects. In contrast, tumor growth and angiopoiesis were not altered by VP22 alone.

In summary, VP22 alone does not directly exert antitumor activity, but mediates the intercellular delivery of PTEN, thereby causing increased number of cells containing PTEN, which results in cells achieving a therapeutic steady state. This leads to an overall increase in the antitumor activity of PTEN, which is correlated with increased antiproliferative effects, cell cycle arrest at G1, induction of apoptosis and antiangiogenic effects. Therefore, our findings show that VP22-mediated intercellular delivery of PTEN enhances the antitumor effects of the latter, providing further experimental data for enhancing the efficacy of PTEN-based gene therapy against cancer. In future studies, we plan to focus on the development of targeting delivery systems based on PTEN-VP22 gene therapy and assess the safety of these gene therapies.

\section{Acknowledgements}

The present study was supported by grants from the National Natural Science Foundation of China (81102288) and Chongqing Science and Technology Commission (cstc2014jcyjA10009).

\section{References}

1. Enzinger PC and Mayer RJ: Esophageal cancer. N Engl J Med 349: 2241-2252, 2003.

2. Pennathur A, Gibson MK, Jobe BA and Luketich JD: Oesophageal carcinoma. Lancet 381: 400-412, 2013.

3. Kamangar F, Dores GM and Anderson WF: Patterns of cancer incidence, mortality, and prevalence across five continents: Defining priorities to reduce cancer disparities in different geographic regions of the world. J Clin Oncol 24: 2137-2150, 2006.

4. Terakawa N, Kanamori Y and Yoshida S: Loss of PTEN expression followed by Akt phosphorylation is a poor prognostic factor for patients with endometrial cancer. Endocr Relat Cancer 10: 203-208, 2003.

5. Kondo Y, Hollingsworth EF and Kondo S: Molecular targeting for malignant gliomas (Review). Int J Oncol 24: 1101-1109, 2004.

6. Koksal IT, Dirice E, Yasar D, Sanlioglu AD, Ciftcioglu A, Gulkesen KH, Ozes NO, Baykara M, Luleci G and Sanlioglu S: The assessment of PTEN tumor suppressor gene in combination with Gleason scoring and serum PSA to evaluate progression of prostate carcinoma. Urol Oncol 22: 307-312, 2004.

7. Im SA, Lee KE, Nam E, Kim DY, Lee JH, Han HS, Seoh JY, Park HY, Cho MS, Han WS, et al: Potential prognostic significance of p185(HER2) overexpression with loss of PTEN expression in gastric carcinomas. Tumori 91: 513-521, 2005.

8. Tang JM, He QY, Guo RX and Chang XJ: Phosphorylated Akt overexpression and loss of PTEN expression in non-small cell lung cancer confers poor prognosis. Lung Cancer 51: 181-191, 2006.

9. Toft DJ and Cryns VL: Minireview: Basal-like breast cancer: from molecular profiles to targeted therapies. Mol Endocrinol 25: 199-211, 2011

10. Tachibana M, Shibakita M, Ohno S, Kinugasa S, Yoshimura H, Ueda S, Fujii T, Rahman MA, Dhar DK and Nagasue N: Expression and prognostic significance of PTEN product protein in patients with esophageal squamous cell carcinoma. Cancer 94: 1955-1960, 2002.

11. Li P, Mao WM, Zheng ZG, Dong ZM and Ling ZQ: Downregulation of PTEN expression modulated by dysregulated miR-21 contributes to the progression of esophageal cancer. Dig Dis Sci 58: 3483-3493, 2013.
12. Sun Z, Ji N, Bi M, Zhang Z, Liu X and Wang Z: Negative expression of PTEN identifies high risk for lymphatic-related metastasis in human esophageal squamous cell carcinoma. Oncol Rep 33: 3024-3032, 2015.

13. Zhao H, Yang J, Fan T, Li S and Ren X: RhoE functions as a tumor suppressor in esophageal squamous cell carcinoma and modulates the PTEN/PI3K/Akt signaling pathway. Tumour Biol 33: 1363-1374, 2012.

14. Ou Y, Ma L, Ma L, Huang Z, Zhou W, Zhao C, Zhang B, Song Y, Yu C and Zhan Q: Overexpression of cyclin B1 antagonizes chemotherapeutic-induced apoptosis through PTEN/Akt pathway in human esophageal squamous cell carcinoma cells. Cancer Biol Ther 14: 45-55, 2013.

15. Sun MM, Zhang MZ, Chen Y, Li SL, Zhang W, Ya GW and Chen KS: Effect of PTEN antisense oligonucleotide on oesophageal squamous cell carcinoma cell lines. J Int Med Res 40: 2098-2108, 2012.

16. Zhou YA, Zhang T, Zhao JB, Wang XP, Jiang T, Gu ZP, Wang XN and Li XF: The adenovirus-mediated transfer of PTEN inhibits the growth of esophageal cancer cells in vitro and in vivo. Biosci Biotechnol Biochem 74: 736-740, 2010.

17. Wills KN, Atencio IA, Avanzini JB, Neuteboom S, Phelan A, Philopena J, Sutjipto S, Vaillancourt MT, Wen SF, Ralston RO, et al: Intratumoral spread and increased efficacy of a p53-VP22 fusion protein expressed by a recombinant adenovirus. J Virol 75: 8733-8741, 2001

18. Zavaglia D, Favrot MC, Eymin B, Tenaud C and Coll JL: Intercellular trafficking and enhanced in vivo antitumour activity of a non-virally delivered P27-VP22 fusion protein. Gene Ther 10: 314-325, 2003.

19. Jin G, Zhou Y, Chai Q, Zhu G, Xu F and Liu F: VP22 and cytosine deaminase fusion gene modified tissue-engineered neural stem cells for glioma therapy. J Cancer Res Clin Oncol 139: 475-483, 2013.

20. Nishikawa M, Otsuki T, Ota A, Guan X, Takemoto S, Takahashi Y and Takakura Y: Induction of tumor-specific immune response by gene transfer of Hsp70-cell-penetrating peptide fusion protein to tumors in mice. Mol Ther 18: 421-428, 2010.

21. Yu X, Xu Z, Lei J, Li T and Wang Y: VP22 mediates intercellular trafficking and enhances the in vitro antitumor activity of PTEN. Mol Med Rep 12: 1286-1290, 2015.

22. Regberg J, Srimanee A and Langel U: Applications of cell-penetrating peptides for tumor targeting and future cancer therapies. Pharmaceuticals (Basel) 5: 991-1007, 2012.

23. Kueltzo LA, Normand N, O'Hare P and Middaugh CR: Conformational lability of herpesvirus protein VP22. J Biol Chem 275: 33213-33221, 2000.

24. Elliott $G$ and $O^{\prime}$ Hare $P$ : Intercellular trafficking and protein delivery by a herpesvirus structural protein. Cell 88: 223-233, 1997.

25. Persad S, Attwell S, Gray V, Delcommenne M, Troussard A, Sanghera J and Dedhar S: Inhibition of integrin-linked kinase (ILK) suppresses activation of protein kinase B/Akt and induces cell cycle arrest and apoptosis of PTEN-mutant prostate cancer cells. Proc Natl Acad Sci USA 97: 3207-3212, 2000.

26. Li Z, Liu GX, Liu YL, Chen X, Huang XL and Gan HT: Effect of adenovirus-mediated PTEN gene on ulcerative colitis-associated colorectal cancer. Int J Colorectal Dis 28: 1107-1115, 2013.

27. Maehama T and Dixon JE: The tumor suppressor, PTEN/ MMAC1, dephosphorylates the lipid second messenger, phosphatidylinositol 3,4,5-trisphosphate. J Biol Chem 273: 13375-13378, 1998.

28. Cully M, You H, Levine AJ and Mak TW: Beyond PTEN mutations: The PI3K pathway as an integrator of multiple inputs during tumorigenesis. Nat Rev Cancer 6: 184-192, 2006.

29. Leslie NR, Yang X, Downes CP and Weijer CJ: PtdIns $(3,4,5)$ $\mathrm{P}(3)$-dependent and -independent roles for PTEN in the control of cell migration. Curr Biol 17: 115-125, 2007.

30. Schwock J, Dhani N and Hedley DW: Targeting focal adhesion kinase signaling in tumor growth and metastasis. Expert Opin Ther Targets 14: 77-94, 2010.

31. Sieg DJ, Hauck CR, Ilic D, Klingbeil CK, Schaefer E, Damsky CH and Schlaepfer DD: FAK integrates growth-factor and integrin signals to promote cell migration. Nat Cell Biol 2: 249-256, 2000. 